\title{
Consistency of capillary wave theory in three dimensions: Divergence of the interface width and agreement with density functional theory
}

John D. Weeks, Wim van Saarloos, Dick Bedeaux, and Edgar Blokhuis

Citation: The Journal of Chemical Physics 91, 6494 (1989); doi: 10.1063/1.457365

View online: https://doi.org/10.1063/1.457365

View Table of Contents: http://aip.scitation.org/toc/jcp/91/10

Published by the American Institute of Physics

\section{Articles you may be interested in}

Structure and thermodynamics of the liquid-vapor interface

The Journal of Chemical Physics 67, 3106 (1977); 10.1063/1.435276

Correlation functions in the capillary wave model of the liquid-vapor interface

The Journal of Chemical Physics 82, 972 (1985); 10.1063/1.448474

Capillary waves and the mean field theory of interfaces

The Journal of Chemical Physics 67, 3636 (1977); 10.1063/1.435301

Capillary waves in a colloid-polymer interface

The Journal of Chemical Physics 122, 134905 (2005); 10.1063/1.1866072

Capillary waves at the liquid-vapor interface and the surface tension of water

The Journal of Chemical Physics 125, 014702 (2006); 10.1063/1.2209240

Molecular dynamics simulations of vapor/liquid coexistence using the nonpolarizable water models

The Journal of Chemical Physics 134, 124708 (2011); 10.1063/1.3574038

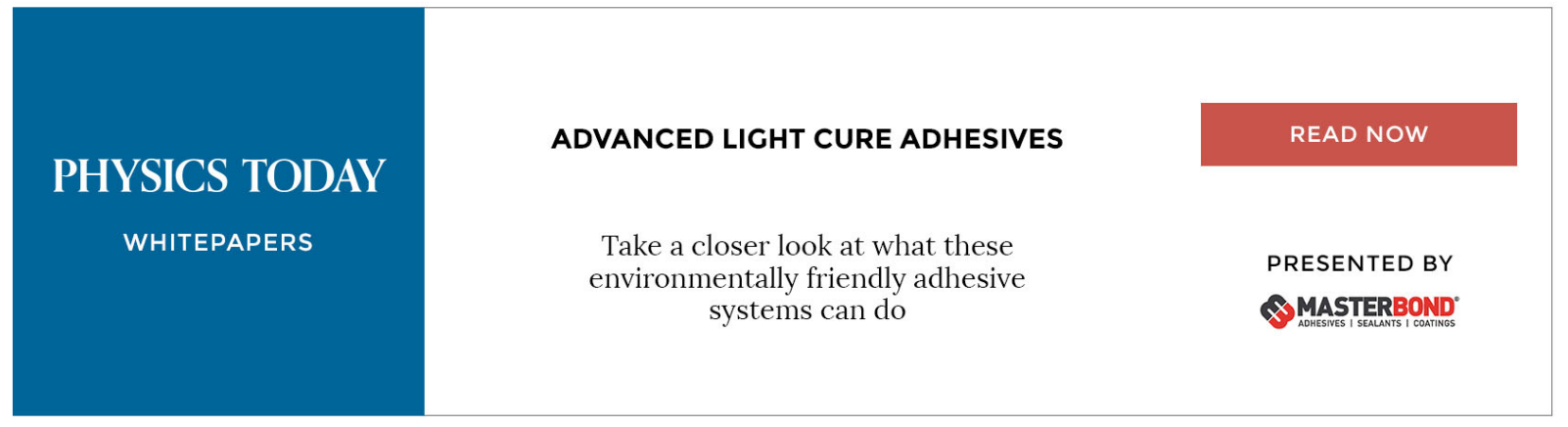




\title{
Consistency of capillary wave theory in three dimensions: Divergence of the interface width and agreement with density functional theory
}

\author{
John D. Weeks and Wim van Saarloos \\ AT\&T Bell Laboratories, Murray Hill, New Jersey 07974 \\ Dick Bedeaux and Edgar Blokhuis \\ Gorlaeus Laboratory, University of Leiden, 2300 RA Leiden, The Netherlands
}

(Received 14 July 1989; accepted 4 August 1989)

\begin{abstract}
We compare the predictions of the capillary wave model for fluid interfaces with known exact expressions, due to Triezenberg, Zwanzig, and Wertheim, that relate the surface tension to the interfacial structure, and find agreement in all dimensions. It is shown that contrary to the case $d<3$, the interfacial pair correlation function does not obey scaling in $d=3$ dimensions on length scales less than the capillary length $L_{c}$. Nevertheless, once the sensitive dependence on the gravitational field and the short distance cutoff is properly taken into account, we find no evidence for recent allegations that the capillary wave model leads to inherently inconsistent results for the direct correlation function in $d=3$. Several issues regarding the connection with density functional theory and the interpretation of the Triezenberg-Zwanzig formula, on which these results touch, are discussed.
\end{abstract}

\section{INTRODUCTION}

Recently Ciach ${ }^{1}$ and Requardt and Wagner ${ }^{2}$ have raised questions about the validity of the Triezenberg-Zwanzig (TZ) formula ${ }^{3}$ for the surface tension $\sigma$ of a planar liquidvapor interface in the particularly relevant case of $d=3 \mathrm{di}$ mensions. They used the capillary wave model ${ }^{4-6}$ to calculate the second moment of the direct correlation function, which appears in the TZ formula [see Eq. (3.10) below], and claimed that the result was divergent or ill-defined for $d=3$. This led to criticism of the density functional formalism $^{7-9}$ used to derive the $\mathrm{TZ}$ formula and to questions about the validity of the conventional capillary wave model as well. A breakdown of the capillary wave picture would indeed have far-reaching consequences, both for general theories of interfacial properties ${ }^{7}$ as well as in detailed treatments of wetting ${ }^{10}$ and roughening transitions. ${ }^{11}$ For example, Requardt and Wagner have suggested that liquid-vapor interfaces may not be "rough" for $d=3$, i.e., may not have a diverging interface width as the external field strength tends to zero.

However, we believe that these concerns are unfounded. A general derivation of the $\mathrm{TZ}$ formula and discussion of some of the broader issues is given elsewhere. ${ }^{12}$ Here we further analyze the capillary wave model in the light of the objections that have been raised. We follow in detail the treatment of the capillary wave model given by Bedeaux and Weeks. ${ }^{6}$ While their basic formalism is valid for arbitrary $d$, the simple scaling analysis they carried out is applicable only for $d<3$. The case $d=3$ represents a crossover dimension below which interface fluctuations are sufficiently strong to produce a diverging interface width $W$ as the external field strength tends to zero, while for $d>3$ the interface width remains finite. As would be expected, a more careful treatment is required for $d=3$, and this calculation is one of the objects of this paper.

While there are several other technical points where we disagree with the analysis of Ciach and of Requardt and
Wagner, we argue that their basic error lies in an inadequate treatment of effects arising from the existence of a finite external field. Capillary wave theory explicitly considers a nonzero external gravitational field ${ }^{13} \phi_{0}(z)=m g z$, which produces macroscopic phase separation in an infinite system for any field strength $g>0$. A further consequence is the existence of a field-dependent length scale, the capillary length $L_{c}$, where

$$
L_{c} \equiv[\sigma / m g \Delta \rho]^{1 / 2},
$$

on which there is exponential decay of the pair correlation function $H$ in the interfacial region. ${ }^{6}$ Here $\sigma$ is the macroscopic surface tension and $\Delta \rho=\rho_{l}-\rho_{v}$ the density difference between the two bulk phases. We assume that the temperature is sufficiently far below the critical temperature $T_{c}$ that $\sigma$ and $\Delta \rho$ can be treated as finite positive constants, and that $g$ is small enough that $L_{c} \gg \xi_{B}$, with $\xi_{B}$ the bulk correlation length. If this decay of the pair correlation function is taken into account, then its inverse function, the direct correlation function $C$ is well-defined and the anomalies found by Ciach and by Requardt and Wagner disappear. A nonzero field is required more generally in order to describe a two-phase system using the grand ensemble, ${ }^{5}$ and to satisfy a basic assumption of the density functional formalism leading to the $\mathrm{TZ}$ formula-the existence of an invertible relation between the external field and the density.

As has been stressed many times, the most convenient way to think about capillary wave theory is in the limit $g \rightarrow 0^{+}$, i.e., $g$ arbitrarily small but nonzero. ${ }^{6,14,15} \mathrm{We}$ will generally work out results to dominating order in this limit, which implies that $L_{c}$ and (for $d \leqslant 3$ ) $W$ are arbitrarily large but finite. There are profound physical and mathematical differences ${ }^{12}$ between this case and the degenerate case with $g \equiv 0$. This sensitive dependence on the external field strength is actually a general property of systems where twophase coexistence is possible. Indeed, the fact that finite but 
arbitrarily weak external fields continue to play an essential role as the thermodynamic limit is taken is often used in the rigorous mathematical theory of phase transitions as a signature of two-phase coexistence. ${ }^{16,17} \mathrm{~A}$ familiar example involving bulk phases at coexistence is the Ising model below $T_{c}$. A finite magnetization is found in the presence of an arbitrarily small external field, while in the complete absence of any symmetry-breaking field the (ensemble-averaged) magnetization vanishes by symmetry. The fact that these two limits do not coincide is obvious physically and causes no problems in the mathematical analysis. Similarly, the thermodynamic limit should be taken at finite $g$ in capillary wave theory.

The plan of this paper is as follows. In Secs. II and III, we first summarize some of the results for the capillary wave model obtained previously by Bedeaux and Weeks ${ }^{6}$ (BW) and report some new results that follow in a straightforward manner. The capillary wave model requires a short-distance "ultraviolet" cutoff to be well-defined, and in Secs. III and IV we discuss the sensitivity of our results to the choice of the cutoff. This issue becomes particularly important for $d=3$. We find that most features of the (long-ranged) pair correlation function $H$ are independent of the cutoff. On the other hand, the inverse of $H$, the direct correlation function $C$, has several features that do depend on the short-ranged behavior of the model, e.g., whether a sharp or smooth cutoff is used in the capillary wave Hamiltonian. We analyze in Sec. IV the scaling behavior ${ }^{6,14}$ of the pair correlation function $H$. As we shall see, for dimension $d<3, H$ assumes a scaling form on all length scales larger than the cutoff, whereas in $d=3$, scaling is found only on length scales that diverge as the capillary length $L_{c}$ tends to infinity. In Secs. V and VI, we turn to an explicit evaluation of $C$ in $d=3$ and to a discussion of the issues raised by Ciach, ${ }^{1}$ and by Requardt and Wagner. ${ }^{2}$ Although we can only obtain $C$ approximately in $d=3$, our results indicate that consistent behavior is found when external field effects are taken into account. In Sec. VII we conclude with a discussion of some of the broader issues this work touches on.

\section{REVIEW OF CAPILLARY WAVE THEORY}

We assume that the reader is familiar with the treatment of $\mathrm{BW}^{6}$ and so we will be brief in our discussion here. For simplicity, we consider an infinite system in the presence of a finite gravitational field $\phi_{0}(z)=m g z$, so that the continuum limit can be taken. Our coordinate system has been chosen so the Gibbs dividing surface is at the $z=0$ plane. After a partial integration over short wavelength degrees of freedom, ${ }^{5,6,18}$ the interface Hamiltonian controlling long wavelength interface distortions can be determined from thermodynamic arguments. It has contributions from the change in area of the distorted surface times the surface tension and from work against the external gravitational field. ${ }^{4}$ If we represent the vertical displacement of the distorted Gibbs dividing surface at position $\mathbf{r}$ in the $z=0$ plane by $z=h(\mathbf{r})$, the interface Hamiltonian for small distortions with $\nabla h(\mathbf{r}) \ll 1$ can be written as

$$
\begin{aligned}
H_{c w}[\{\hat{h}(\mathbf{q})\}]= & \frac{\sigma}{2}\left(\frac{1}{2 \pi}\right)^{d-1} \\
& \times \int_{|q|<q_{\max }} d \mathbf{q} \hat{h}(\mathbf{q}) \hat{h}(-\mathbf{q})\left[q^{2}+L_{c}^{-2}\right],
\end{aligned}
$$

where $\hat{h}(q)$ is the Fourier transform of the interface distortion $h(\mathbf{r})$ :

$$
\hat{h}(\mathbf{q}) \equiv \int d \mathbf{r} h(\mathbf{r}) e^{-i \mathbf{q r} r},
$$

and $\mathbf{r}$ and $\mathbf{q}$ are $d-1$ dimensional vectors. The coarse graining over short wavelength degrees of freedom leads to the simple single-valued interface Hamiltonian (2.1) when fluctuations up to $O\left(\xi_{B}\right)$ are integrated out. ${ }^{5,6,18}$ The interface Hamiltonian can consistently describe the remaining long wavelength interface degrees of freedom provided that the integration in Eq. (2.1) is restricted to wave vectors $|q| \leqslant q_{\max } \propto \xi_{B}^{-1}$. This "sharp cutoff" is simple to treat mathematically, but one must keep in mind that it can introduce some artifical features, ${ }^{19}$ especially at small $r$. Whenever possible, the short-distance behavior at "microscopic" scales $r \lesssim q_{\max }^{-1}$ calculated using Eq. (2.1) is taken to be a smooth extrapolation of the behavior seen on larger scales. Results depending specifically on the value of the cutoff are likely to be artifacts of this approximation. We can expect universal (model independent) behavior only for properties independent of the cutoff that are dominated by long wavelength fluctuations, where the thermodynamic arguments leading to Eq. (2.1) are valid.

A fundamental measure of interface fluctuations is given by the height-height correlation function $S(r)$ $\equiv\langle h(\mathbf{r}+\mathbf{s}) h(\mathbf{s})\rangle$, where \langle\rangle denotes an ensemble average taken using the interface Hamiltonian (2.1). We have then

$$
S(r)=\frac{1}{\beta \sigma(2 \pi)^{d-1}} \int_{|q|<q_{\max }} d \mathbf{q} \frac{e^{i \mathbf{q} \cdot r}}{\left(q^{2}+L_{c}^{-2}\right)} .
$$

Here $\beta=\left(k_{B} T\right)^{-1}$, with $T$ the temperature. The mean squared height fluctuations give a measure of the interface width $W$, defined as

$$
W^{2} \equiv S(0),
$$

and we also define the height difference correlation function $^{5,14}$

$G(r) \equiv \frac{1}{2}\left\langle[h(\mathbf{r}+\mathbf{s})-h(\mathbf{s})]^{2}\right\rangle=W^{2}-S(r)$.

Clearly $G(0)=0$ and $G(\infty)=W^{2}$. More detailed information about interface height correlations is given by the singlet height distribution function $p(z) \equiv\langle\delta[h(\mathbf{r})-z]\rangle$ and the pair function $P\left(z_{1}, z_{2}, r_{12}\right) \equiv\left\langle\delta\left[h\left(\mathbf{r}_{1}\right)-z_{1}\right] \delta\left[h\left(\mathbf{r}_{2}\right)\right.\right.$ $\left.\left.-z_{2}\right]\right\rangle$. BW find

$$
p\left(z_{1}\right)=\left[2 \pi W^{2}\right]^{-1 / 2} \exp \left[-\frac{z^{2}}{2 W^{2}}\right],
$$

and, in a particularly convenient representation,

$P\left(z_{1}, z_{2}, r_{12}\right)=\exp \left[S\left(r_{12}\right) \frac{\partial^{2}}{\partial z_{1} \partial z_{2}}\right] p\left(z_{1}\right) p\left(z_{2}\right)$,

where the exponential operator is defined by its Taylor series expansion. 
Density fluctuations are related to interface height fluctuations by the assumption that the local coarse-grained density at some point $\mathbf{R}=(\mathbf{r}, z)$ in a particular configuration of heights $\{h\}$ is the bulk vapor (liquid) density provided the point is located above (below) the distorted surface whose location is given by $h(r)$. Thus the local "density operator" is

$$
\rho_{\mathrm{op}}(\mathbf{R},\{h\}) \equiv \frac{1}{2}\left(\rho_{l}+\rho_{v}\right)+\frac{1}{2} \Delta \rho \operatorname{sgn}[h(r)-z],
$$

and the average density profile $\rho(z)=\left\langle\rho_{\text {op }}(\mathbf{R},\{h\})\right\rangle$ satisfies the equation

$$
\frac{d \rho(z)}{d z} \equiv \rho^{\prime}(z)=-\Delta \rho p(z)
$$

or

$$
\rho(z)=\frac{1}{2}\left(\rho_{l}+\rho_{v}\right)-\frac{1}{2} \Delta \rho \operatorname{erf}\left(\frac{z}{\sqrt{2} W}\right) .
$$

Similarly, the pair correlation function

$$
\begin{aligned}
H\left(z_{1}, z_{2}, r_{12}\right) \equiv & \left\langle\left[\rho_{\mathrm{op}}\left(\mathbf{R}_{1},\{h\}\right)-\rho\left(z_{1}\right)\right]\right. \\
& \left.\times\left[\rho_{\mathrm{op}}\left(\mathbf{R}_{2},\{h\}\right)-\rho\left(z_{2}\right)\right]\right\rangle
\end{aligned}
$$

is easily seen from Eqs. (2.7)-(2.9) to satisfy the equation

$$
\begin{aligned}
H\left(z_{1}, z_{2}, r_{12}\right)= & \left\{\exp \left[S\left(r_{12}\right) \frac{\partial^{2}}{\partial z_{1} \partial z_{2}}\right]-1\right\} \\
& \times \rho\left(z_{1}\right) \rho\left(z_{2}\right) .
\end{aligned}
$$

Expanding Eq. (2.11) and noting that derivatives of $\rho(z)$ vanish far from the interface we find that on integration over $z_{2}$ only the linear term survives:

$\int d z_{2} H\left(z_{1}, z_{2}, r_{12}\right)=-S\left(r_{12}\right) \Delta \rho d \rho\left(z_{1}\right) / d z_{1}$.

For completeness we mention two other exact representations of $H$ that can easily be derived from the results of BW. Differentiating Eq. (2.11) with respect to $z_{1}$ and $z_{2}$, we note that the result can be written in the form of Eq. (2.11) with $\rho(z)$ replaced by $\rho^{\prime}(z)$. Defining the Fourier transform in the vertical direction by

$$
\tilde{f}(k)=\int_{-\infty}^{\infty} d z f(z) e^{-i k z},
$$

and using Eqs. (2.6) and (2.9), we find on taking transforms

$$
\begin{aligned}
\widetilde{H}\left(k_{1}, k_{2}, r_{12}\right)= & (\Delta \rho)^{2} e^{1 / 2 W^{2}\left(k_{1}^{2}+k_{2}^{2}\right)} \\
& \times\left[1-e^{-k_{1} k_{2} S\left(r_{12}\right)}\right] / k_{1} k_{2} .
\end{aligned}
$$

Finally, comparing Eq. (2.11) differentiated with respect to $r_{12}$ and to $z_{1}$ and $z_{2}$, and using Eqs. (3.9) and (3.11) of BW yields

$$
\begin{aligned}
H\left(z_{1}, z_{2}, r_{12}\right)= & \frac{(\Delta \rho)^{2}}{2 \pi} \int_{0}^{\arcsin \left[S\left(r_{12}\right) / W^{2}\right]} d \alpha \\
& \times \exp \left[\frac{-\left(z_{1}^{2}+z_{2}^{2}\right)+2 z_{1} z_{2} \sin \alpha}{2 W^{2} \cos ^{2} \alpha}\right] .
\end{aligned}
$$

For $z_{1}=z_{2}=0$, we have

$$
H\left(0,0, r_{12}\right)=\frac{(\Delta \rho)^{2}}{2 \pi} \arcsin \left[S\left(r_{12}\right) / W^{2}\right],
$$

which is equivalent to Eq. (4.8) of BW. These equations are exact for the capillary wave model and hold in all dimensions.

\section{GENERAL RESULTS}

We first show that the modified version of the TZ formula for the surface tension derived by Wertheim, ${ }^{20}$ which we call the $T Z W$ formula,

$$
\beta \sigma=\frac{(\beta m g)^{2}}{2(d-1)} \int d \mathbf{r}_{12} \int d z_{1} \int d z_{2} r_{12}^{2} H\left(z_{1}, z_{2}, r_{12}\right),
$$

is satisfied as an identity in capillary wave theory in all dimensions. Using Eq. (2.12) in Eq. (3.1) we have

$$
\beta \sigma=\frac{(\beta m g)^{2}}{2(d-1)}(\Delta \rho)^{2} \int d \mathbf{r}_{12} r_{12}^{2} S\left(r_{12}\right)
$$

or

$$
\beta \sigma=\left.\frac{(\beta m g)^{2}}{2(d-1)} \frac{\partial}{\partial \mathbf{q}} \cdot \frac{\partial}{\partial \mathbf{q}} \hat{S}(q)\right|_{q=0},
$$

where, from Eq. (2.3), for small $q$,

$$
\begin{aligned}
\widehat{S}(q) & \equiv \int d \mathbf{r} S(r) e^{-i q \cdot \mathbf{r}} \\
& =\frac{1}{\beta \sigma\left[q^{2}+L_{c}^{-2}\right]} .
\end{aligned}
$$

Using Eq. (1.1), we see that Eq. (3.3) is indeed satisfied identically. This result only involves properties of $\widehat{S}(q)$ for $q$ near zero and is independent of the cutoff at $q=q_{\max }$. Hence it also should hold for any microscopic model which may differ from the capillary wave model on small length scales but which satisfies Eq. (3.4) for arbitrarily long wavelength distortions.

Note that Eq. (3.1) is satisfied identically provided that the macroscopic $\sigma$ and not some "bare" $\sigma_{B}$ is used in the interface Hamiltonian (2.1).$^{5,6,14,18,19}$ Strictly speaking, it is only for arbitrarily long wavelength distortions that the simple quadratic Hamiltonian (2.1) is correct; such distortions are clearly controlled by the macroscopic $\sigma$. Technically, in order to reproduce this limit correctly, we must make such a choice for $\sigma$ since the coupling constant in a Gaussian Hamiltonian does not get renormalized. Alternatively, we can think of Eq. (3.1) as a "sum rule" that fixes $\sigma_{B}=\sigma$ in the simple capillary wave model. In the interpretation of Weeks, ${ }^{5}$ the quadratic model with $\sigma_{B}=\sigma$ then holds as a good approximation for shorter wavelength distortions, even down to distortions with wavelengths of $O\left(\xi_{B}\right)$.

Note also that capillary wave theory does not give a prescription for actually calculating the surface tension; rather $\sigma$ is a parameter appearing in the interface Hamiltonian. Thus Eq. (3.1) is best thought of as a consistency condition which correlation functions calculated from the theory must obey.

An exact treatment of interface distortions approximately described by Eq. (2.1) could in principle be carried out using renormalization group methods. ${ }^{21}$ For shorter wavelength distortions there must be interactions between the "normal modes" given in Eq. (2.1); these could be reex- 
pressed using a wave-vector dependent $\sigma(q)$ in Eq. (2.1), with only the long wavelength limit $\sigma(q \rightarrow 0)$ given by the macroscopic value. Kayser ${ }^{22}$ and van Leeuwen and Sengers ${ }^{23}$ have considered interesting phenomenological extensions of the capillary wave model based on this idea. Here, however, we continue to use the simple quadratic model with $\sigma_{B}=\sigma$ as an approximate representation of the shorter wavelength distortions, but attribute universal significance only to those results, such as Eq. (3.3), which are independent of the cutoff and dominated by arbitrarily long wavelength fluctuations.

Most of the controversy has involved properties of the (generalized) direct correlation function $C$, which is defined as the inverse of $H$ :

$$
\begin{gathered}
\int d z_{3} \int d \mathbf{r}_{3} H\left(z_{1}, z_{3}, r_{13}\right) C\left(z_{3}, z_{2}, r_{32}\right) \\
=\delta\left(z_{1}-z_{2}\right) \delta\left(\mathbf{r}_{1}-\mathbf{r}_{2}\right) .
\end{gathered}
$$

While a calculation of $C$ in $d=3$ has some subtle features that are discussed in the next sections, it is easy to show that the TZ formula is satisfied exactly in all $d$. Multiplying Eq. (2.12) by $C\left(z_{3}, z_{1}, r_{13}\right)$ and integrating over $\mathbf{R}_{1}=\left(\mathbf{r}_{1}, z_{1}\right)$ yields from Eq. (3.5)

$$
\begin{aligned}
& -\Delta \rho \int d z_{1} \int d \mathbf{r}_{1} C\left(z_{3}, z_{1}, r_{31}\right) \rho^{\prime}\left(z_{1}\right) S\left(r_{12}\right) \\
& \quad=\delta\left(\mathbf{r}_{3}-\mathbf{r}_{2}\right),
\end{aligned}
$$

or, taking Fourier transforms,

$$
\begin{aligned}
\int d z_{3} \widehat{C}\left(z_{1}, z_{3}, q\right) \rho^{\prime}\left(z_{3}\right) & =-[\Delta \rho \hat{S}(q)]^{-1} \\
& =\frac{-\beta \sigma}{\Delta \rho}\left[q^{2}+L_{c}^{-2}\right],
\end{aligned}
$$

using Eq. (3.4). Formally taking inverse transforms gives

$$
\begin{aligned}
\int d z_{3} C\left(z_{1}, z_{3}, r_{13}\right) \rho^{\prime}\left(z_{3}\right)= & \frac{-\beta \sigma}{\Delta \rho L_{c}^{2}} \delta\left(\mathbf{r}_{1}-\mathbf{r}_{3}\right) \\
& +\frac{\beta \sigma}{\Delta \rho} \nabla^{2} \delta\left(\mathbf{r}_{1}-\mathbf{r}_{3}\right)
\end{aligned}
$$

Since the $\delta$ functions in the definition of the inverse in Eq. (3.5) directly involve short-distance behavior, we expect that some features of $C$ will depend on the choice of the cutoff. Thus, strictly speaking, the $\delta$ functions in Eq. (3.8) should be understood as $\delta$ functions on scales larger than the microscopic scale $q_{\max }^{-1}$, since our use of the cutoff for $q>q_{\max }$ does not allow a precise determination of the microscopic scale. That is, when integrated over $r_{3}$ the zeroth and second moments computed from Eq. (3.8) are correctly given by treating the functions as true $\delta$ functions, in exact agreement with the moments computed from the more fundamental Eq. (3.7). Note that the values of the zeroth and second moments so calculated from Eq. (3.7) or (3.8) rely on Eq. (3.4) for small $q$ only, and are independent of the cutoff. As shown below, both are in complete agreement with exact identities. On the other hand, the apparent strict vanishing of all higher order moments in Eq. (3.7b) depends explicitly on the assumption of a sharp cutoff, so this prediction cannot be universal. A microscopic model with a differ- ent treatment of the cutoff could have some additional structure on the short length scale, and different values for higher moments. ${ }^{24}$

These equations show that $C$ has very simple properties when "projected" into $\rho^{\prime}(z)$ as in Eq. (3.7) or (3.8)..$^{12}$ It is precisely this projected combination that appears in the formula

$$
-\beta m g=\int d z_{3} \int d \mathbf{r}_{3} C\left(z_{1}, z_{3}, r_{13}\right) \rho^{\prime}\left(z_{3}\right),
$$

first derived by Wertheim ${ }^{20}$ and by Lovett et $a l .{ }^{9}$ and in the original $\mathrm{TZ}$ formula ${ }^{3}$ for the surface tension

$$
\begin{aligned}
\beta \sigma= & -\frac{1}{2(d-1)} \int d z_{1} \int d z_{2} \int d \mathbf{r}_{2} r_{12}^{2} \rho^{\prime}\left(z_{1}\right) \\
& \times C\left(z_{1}, z_{2}, r_{12}\right) \rho^{\prime}\left(z_{2}\right) .
\end{aligned}
$$

Using Eqs. (1.1) and (3.7) or Eq. (3.8) we see that Eqs. (3.9) and (3.10) are again satisfied as identities in the capillary wave model. In particular, the capillary wave model (in all dimensions) is consistent with the TZ formula (3.10). This is not surprising since capillary wave theory is based on the same fundamental idea that is used to derive the $\mathrm{TZ}$ formula: the change in free energy for long wavelength interface distortions is given by the macroscopic surface tension times the change in area.

Stecki and co-workers ${ }^{24,25}$ have defined a "conditional" direct correlation function in terms of the inverse of $\boldsymbol{P}$ in Eq. (2.7), which is supposed to have especially simple properties. They argued that it is accurately approximated by $C\left(z_{1}, z_{2}, r_{12}\right) \rho^{\prime}\left(z_{2}\right)$. Equation (3.7) or (3.8) are exact results (within the capillary wave model) for the integral over $z_{2}$ of this combination of functions, and we see that the result is short-ranged in $r_{12}$.

Note that Eq. (3.1) holds true precisely because $g>0$, so that the second moment of $H$ exists. This same requirement is implicit in Eq. (3.10), in order that the inverse function $C$ is well-defined. To argue that the second moment of $C$ does not exist under these conditions ${ }^{1,2}$ requires that $C$ is even longer-ranged than $H$ ! This is certainly not the case for the projection of $C$ given in Eq. (3.7) or (3.8). In the next sections we turn to a direct calculation of $C$, concentrating on the contentious case $d=3$. We first discuss attempts to apply scaling concepts used for $d<3$ to the case of $d=3$, where we disagree with several conclusions of Ciach and Requardt and Wagner.

\section{SCALING BEHAVIOR OF $H$}

\section{A. Scaling theory for $d<3$}

For $d<3$, BW showed that as $g \rightarrow 0^{+}\left(L_{c} \rightarrow \infty\right), H$ could be put into the scaling form ${ }^{14}$

$$
H\left(z_{1}, z_{2}, r_{12} ; g, q_{\max }\right)=H_{s}\left(\tau_{1}, \tau_{2}, x_{12}\right),
$$

where distances normal to the interface are scaled by the interface width:

$$
\tau \equiv \frac{z}{\sqrt{2} W}
$$

and the transverse distances along the interface scaled by the capillary length $L_{c}$ : 


$$
x_{12} \equiv r_{12} / L_{c} .
$$

We have explicitly indicated on the left-hand side of Eq. (4.1) the dependence of the correlation function $H$ on the two parameters $g$ and $q_{\max }$ in the capillary wave Hamiltonian (2.1). The right-hand side indicates that for $d<3$ the only dependence on $g$ in $H$ to dominant order as $g \rightarrow 0^{+}$is through its effects on the arguments $\tau$ and $x$. In general, the subscript $s$ will be used to denote a scaling function for which the only dependence on $g$ as $g \rightarrow 0^{+}$is through its arguments.

The fact that Eq. (4.1) holds for all $r \gtrsim q_{\max }^{-1}$ for $d<3$ can easily be seen by rewriting Eq. (2.11) as

$$
\begin{aligned}
H\left(z_{1}, z_{2}, r_{12}\right)= & \left\{\exp \left[\frac{1}{2} f\left(r_{12}\right) \frac{\partial^{2}}{\partial \tau_{1} \partial \tau_{2}}\right]-1\right\} \\
& \times \rho_{s}\left(\tau_{1}\right) \rho_{s}\left(\tau_{2}\right),
\end{aligned}
$$

where the normalized height correlation function $f$ is

$$
\begin{aligned}
f\left(r_{12}\right) & \equiv S\left(r_{12}\right) / S(0), \\
& =S\left(r_{12}\right) / W^{2},
\end{aligned}
$$

and $\rho_{s}(\tau)=\rho(z)$ is the right-hand side of Eq. (2.10). Clearly $f\left(r_{12}\right)$ tends to unity for small $r_{12}$ and from Eq. (2.3) it vanishes exponentially for $r_{12} \gtrsim L_{c}$.

For $d<3$ we can ignore the restriction on $q_{\max }$ in Eq. (2.3) as $g \rightarrow 0^{+}$, since the integral converges as $q \rightarrow \infty$ and is dominated by the small $q$ behavior. As usual, the results so obtained will give the behavior to dominant order as $g \rightarrow 0^{+}$. As shown by $\mathrm{BW}, S$ is then a function of the scaled variable $x$ in Eq. (4.3) and $f$ has the scaling form

$$
f\left(r_{12}\right)=f_{s}\left(x_{12}\right) \text {, }
$$

where for small $x$,

$$
f_{s}(x)=1-c x^{3-d}+\cdots
$$

with $c$ a known constant of $O(1)$.

Equation (4.4) together with Eq. (4.6) explicitly shows that $H$ indeed has the scaling form given in Eq. (4.1) on all length scales $r \gtrsim q_{\max }^{-1}$. Contrary to the conclusion reached by Ciach after her Eq. (2.1b), the validity of the scaling form for $H$ is not inconsistent with known results for $P$. Ciach's argument can be seen most easily by rewriting Eq. (2.7) in scaled variables, similar to Eq. (4.4). Using Eqs. (2.9) and (2.10), this suggests that $P$ is $O\left(W^{-2}\right)$. However, the fact that $P\left(z_{1}, z_{2}, r_{12}\right) / p\left(z_{2}\right)$ plays the role of a conditional probability shows that $P$ is actually $O\left(W^{-1}\right)$ for small $r_{12}$. The apparent inconsistency disappears if one realizes that the exponential operator is not necessarily "of order unity." Indeed it is easy to check that Eq. (2.7) leads exactly to $P\left(z_{1}, z_{2}, r_{12}\right) \rightarrow p\left(z_{2}\right) \delta\left(z_{1}-z_{2}\right)$ for $r_{12} \rightarrow 0$, as one would expect intuitively. The "intrinsic structure" discussed by Ciach for small $r_{12}$ is simply that given by the short distance behavior of the height difference correlation function $G(r)$ in Eq. (2.5) for $q_{\max }^{-1} \lesssim r \ll L_{c}$ and this is perfectly consistent with predictions of the scaling analysis, which uses the equivalent function $S(r)$ in Eq. (2.3). Our analysis is more general since we also consider the limiting behavior for $r>L_{c}$ where $S(r) \rightarrow 0$ and $G(r) \rightarrow W^{2}$. To properly describe $d=3$ it is necessary to consider both large scales where $\tau$ and $x$ are $O(1)$ as well as the behavior at shorter distances.

The scaling of $H$ implies a similar scaling form for the inverse function $C$. Defining a scaled direct correlation function $C_{s}$ by

$$
\begin{aligned}
& \int d \tau_{3} \int d \mathbf{x}_{3} H_{s}\left(\tau_{1}, \tau_{3}, x_{13}\right) C_{s}\left(\tau_{3}, \tau_{2}, x_{32}\right) \\
& =\delta\left(\tau_{1}-\tau_{2}\right) \delta\left(\mathbf{x}_{1}-\mathbf{x}_{2}\right),
\end{aligned}
$$

we have on comparing with Eq. (3.5) the small $g$ scaling result

$$
C\left(z_{1}, z_{2}, r_{12} ; g, q_{\max }\right)=\frac{1}{2 L_{c}^{2(d-1)} W^{2}} C_{s}\left(\tau_{1}, \tau_{2}, x_{12}\right) .
$$

As $g \rightarrow 0^{+}$, these are exact results for the capillary wave model for $d<3$. Again, other models with a different treatment of the microscopic cutoff ${ }^{19,24,25}$ will have different (nonuniversal) behavior on the microscopic scale of $O\left(\xi_{B}\right)$.

The fact that $C$ in Eq. (4.9) can be expressed in terms of scaling variables is not inconsistent with $C$ being short ranged, as Eq. (3.8) suggests. Indeed explicit calculations ${ }^{6}$ in $d=2$ show that $C$ can be written exactly in terms of $\delta$ functions of the scaled variables. As we will see, the dominant term in $d=3$ has exactly the same form.

\section{B. Lack of scaling for $d=3$}

For $d=3$ we can no longer ignore the cutoff at $q_{\max }$ in computing $S$ and the simple scaling analysis given above breaks down. ${ }^{26}$ Instead, as shown by BW, we have from Eq. (2.3) as $L_{c} \rightarrow \infty$ in $d=3$

$$
W^{2}=S(0)=\frac{1}{2 \pi \beta \sigma} \ln \left(L_{c} q_{\max }\right) .
$$

Although Eq. (4.10) formally depends on the choice of cutoff $q_{\max }$, its dependence is so weak for $L_{c}$ large that any reasonable choice gives virtually the same result. However, we will keep the formal cutoff dependence in Eq. (4.10), since in other expressions it will become much more significant. Similarly, the height-height correlation function in $d=3$ is $^{6}$

$$
S(r)=\frac{1}{2 \pi \beta \sigma} K_{0}\left[\left(r^{2}+q_{\max }^{-2}\right)^{1 / 2} / L_{c}\right],
$$

where $K_{0}$ is the modified Bessel function of the second kind. Thus for $q_{\max }^{-1} \lesssim r, f(r)$ from Eqs. (4.5) and (4.11) has the form

$$
f(r)=\frac{K_{0}(x)}{2 \pi \beta \sigma W^{2}} \equiv \frac{\bar{f}_{s}(x)}{W^{2}},
$$

where $x \equiv r / L_{c}$. Although Eq. (4.12) shows that $f$ for $r \gtrsim$ $q_{\max }^{-1}$ can be written in terms of the scaling variable $x$, we have put a bar over $\bar{f}_{s}$ in Eq. (4.12) to remind us that $\bar{f}_{s}(x)$ is not $O(1)$ for small $x$, unlike the previous result, Eq. (4.7), which was valid for $d<3$. By definition, $f(r)$ tends to unity for small $r$ so $\bar{f}_{s}(x)$ in Eq. (4.12) tends to $W^{2}$ for small $x$. This singular behavior of the "scaling function" $\bar{f}_{s}$ for $d=3$ must be kept in mind in what follows.

In particular, it is not true that $\bar{f}_{s}(x) / W^{2}$ is uniformly small as $W \rightarrow \infty\left(g \rightarrow 0^{+}\right)$. Rather we find for $q_{\max }^{-1} \leqslant r \ll L_{c}$ in Eq. (4.12) 


$$
f(r)=1-\frac{\ln \left(r q_{\max }\right)}{\ln \left(L_{c} q_{\max }\right)}+\cdots
$$

and for $r \gtrsim L_{c}$

$$
f(r) \approx \frac{(\pi / 2 x)^{1 / 2} e^{-x}}{2 \pi \beta \sigma W^{2}}
$$

Thus $f(r) \approx 1 / 2$ for $r \approx L_{c}^{1 / 2}, f(r) \approx W^{-2}$ for $r \approx L_{c}$, with exponential decay to zero for $r>L_{c}$.

The implications for scaling of $H$ in $d=3$ follow from Eqs. (4.12) and (4.4). For general $r_{12}$ we can conclude only that

$$
H\left(z_{1}, z_{2}, r_{12}\right)=H_{s}\left[\tau_{1}, \tau_{2}, \bar{f}_{s}\left(x_{12}\right) / W^{2}\right] .
$$

However, at very large $r$, when $f(r) \ll 1$, only the linear term in the exponential operator in Eq. (4.4) is important and we then have $H \approx \mathrm{W}^{-2} H_{s}\left(\tau_{1}, \tau_{2}, x_{12}\right)$. This limiting scaling form was assumed to be generally correct by Requardt and Wagner in their Eq. (3.14), which is simply the linear term of our Eq. (4.4):

$$
H \approx \frac{1}{2} \frac{K_{0}\left(x_{12}\right)}{2 \pi \beta \sigma W^{2}} \frac{d \rho_{s}\left(\tau_{1}\right)}{d \tau_{1}} \frac{d \rho_{s}\left(\tau_{2}\right)}{d \tau_{2}} .
$$

Although $f$ can (formally) be put into a scaling form for $q_{\max }^{-1} \leqslant r$, it is only at much larger $r$ (a distance much greater than, say, $L_{c}^{1 / 2}$ ) that $H$ takes on the scaling form assumed by Requardt and Wagner.

Equation (4.16) does give the correct asymptotic behavior for $H$ at these very large separations ${ }^{6}$ and it is the only term in the expansion of $H$ that survives when integrated over $z$ as in Eq. (2.12). Thus the TZW formula (3.1) projects out all parts of $H$ except for the right-hand side of Eq. (4.16). This projected $H$ takes on the Requardt and Wagner scaling form for $q_{\max }^{-1} \leqslant r$, but the full $H$ does so only at much larger $r$.

This slow approach to the asymptotic scaling form in $d=3$ contrasts to that found earlier for $d<3$ and also differs from $d>3 .{ }^{26}$ In the latter case, ${ }^{6,14}$ the asymptotic form $H \approx r^{-\theta} H_{s}\left(\tau_{1}, \tau_{2}, x_{12}\right)$ with $\theta=d-3$ is again valid on scales larger than the cutoff, with corrections to this dominant behavior falling off as $r^{-2 \theta}$. For $r \gtrsim L_{c}, H$ again vanishes exponentially.

\section{CALCULATION OF THE DOMINANT TERM IN $C$ FOR $d=3$}

An exact and closed form calculation of $C$ for the capillary wave model appears to be possible only for $d=2$, as carried out by BW. Here we calculate the dominant term in $C$ for fixed $z_{1}, z_{2} \ll W$ and $r_{12} \ll L_{c}$ in the limit $g \rightarrow 0^{+}$for $d=3$.

Taking Fourier transforms of Eq. (3.5) we have

$$
\int d z_{3} \hat{H}\left(z_{1}, z_{3}, q\right) \hat{C}\left(z_{3}, z_{2}, q\right)=\delta\left(z_{1}-z_{2}\right) \text {. }
$$

Let $\widehat{H}_{\tau}\left(\tau_{1}, \tau_{2}, q\right) \equiv \hat{H}\left(z_{1}, z_{2}, q\right)$ and define $\widehat{C}_{\tau}\left(\tau_{1}, \tau_{2}, q\right)$ by the equation

$\int d \tau_{3} \widehat{H}_{\tau}\left(\tau_{1}, \tau_{3}, q\right) \widehat{C}_{\tau}\left(\tau_{3}, \tau_{2}, q\right)=\delta\left(\tau_{1}-\tau_{2}\right)$.

Comparing with Eq. (5.1) we see that

$$
C_{\tau}\left(\tau_{1}, \tau_{2}, q\right)=2 W^{2} \hat{C}\left(z_{1}, z_{2}, q\right) .
$$

These transformations are just convenient changes of variables and involve no scaling assumptions. In particular, $\widehat{C}_{\tau}\left(\tau_{1}, \tau_{2}, q\right)$ may have some explicit dependence on $g$ in addition to that implied by its dependence on $\tau$.

We now make use of the detailed eigenfunction expansions of BW, which follow directly from Eq. (4.4). Though they assumed the scaling form for $f$ in Eq. (4.6) appropriate for $d<3$, the form of their results holds also for $d=3$ provided we use the (unscaled) $f(r)$ and take transforms using the unscaled wave vector $q$ as in Eq. (5.1). Thus Eq. (6.8) of $\mathrm{BW}$ can be written more generally as

$$
\begin{aligned}
\hat{H}_{\tau}\left(\tau_{1}, \tau_{2}, q\right)= & e^{-1 / 2 \tau_{1}^{2}-1 / 2 \tau_{2}^{2}}(\Delta \rho)^{2} \frac{1}{2 \sqrt{\pi}} \\
& \times \sum_{m=0}^{\infty} \frac{\hat{f}_{m+1}(q)}{(m+1)} \psi_{m}\left(\tau_{1}\right) \psi_{m}\left(\tau_{2}\right),
\end{aligned}
$$

where

$$
\hat{f}_{m}(q) \equiv \int d \mathbf{r}[f(r)]^{m} e^{-i \mathbf{q} \cdot \mathbf{r}},
$$

and $\psi_{m}(\tau)$ is the harmonic oscillator eigenfunction:

$$
\psi_{m}(\tau) \equiv H_{m}(\tau) e^{-1 / 2 \tau^{2}}\left[\sqrt{\pi} 2^{m} m !\right]^{-1 / 2},
$$

with $H_{m}$ a Hermite polynominal. We are also interested in the interface moments

$$
f_{m, 0} \equiv \int d \mathbf{r}[f(r)]^{m}
$$

and

$$
f_{m, 2} \equiv \int d \mathbf{r} r^{2}[f(r)]^{m},
$$

which can be obtained formally from the power series expansion about $q=0$ of Eq. (5.5).

It follows from Eq. (5.4) and the completeness relation for the $\psi_{m}$ that $C_{\tau}$ in Eq. (5.2) is given by

$$
\begin{aligned}
\widehat{C}_{\tau}\left(\tau_{1}, \tau_{2}, q\right)= & e^{1 / 2 \tau_{1}^{2}+1 / 2 \tau_{2}^{2}}(\Delta \rho)^{-2} 2 \sqrt{\pi} \\
& \times \sum_{m=0}^{\infty} \frac{(m+1)}{\hat{f}_{m+1}(q)} \psi_{m}\left(\tau_{1}\right) \psi_{m}\left(\tau_{2}\right) .
\end{aligned}
$$

This is the analog of Eq. (6.10) of BW. These equations hold in all dimensions since no assumptions about the scaling of $f(r)$ have been made.

We now turn to an evaluation of Eq. (5.5) in $d=3$, noting that to dominant order the $q_{\max }^{-2}$ term in Eq. (4.11) can be ignored in the integration over $r$ in Eq. (5.5). Thus Eq. (5.5) can be written for $d=3$ as

$\hat{f}_{m}(q)=\frac{1}{\left(2 \pi \beta \sigma W^{2}\right)^{m}} \int d \mathbf{r} e^{-i \mathbf{q} \cdot r}\left[K_{0}\left(r / L_{c}\right)\right]^{m}$.

We have been able to carry out the integration in (5.10) exactly only for $m=1$ and $m=2$, yielding ${ }^{27}$

$$
\hat{f}_{1}(q)=\frac{L_{\mathrm{c}}^{2}}{\beta \sigma W^{2}\left(1+Q^{2}\right)},
$$

where $Q \equiv q L_{c}$, in agreement with Eq. (3.4), and $\mathrm{d}^{28}$ 
$\hat{f}_{2}(q)=\frac{2 \pi L_{c}^{2}}{\left(2 \pi \beta \sigma W^{2}\right)^{2}} \frac{1}{2\left(u^{2}-1\right)^{1 / 2}} \ln \left[u+\left(u^{2}-1\right)^{1 / 2}\right]$,

where $u \equiv 1+Q^{2} / 2$. Equations (5.11) and (5.12) exhibit very different behaviors, depending on what value of the wave vector $q$ we consider. For any $q \gg L_{c}^{-1}$, and hence any fixed $q>0$ as $L_{c} \rightarrow \infty$, Eqs. (5.11) and (5.12) give to dominant order

$$
\begin{aligned}
\hat{f}_{1}(q) & \approx \frac{1}{\beta \sigma W^{2} q^{2}}, \\
\hat{f}_{2}(q) & \approx \frac{2 \pi L_{c}^{2} \ln Q^{2}}{\left(2 \pi \beta \sigma W^{2}\right)^{2} Q^{2}} \\
& \approx \frac{2}{\beta \sigma W^{2} q^{2}}\left[1+O\left(W^{-2} \ln q\right)\right],
\end{aligned}
$$

where we have used Eq. (4.10). By taking inverse transforms, these approximations can be used to describe the functions $f_{1}(r)$ and $f_{2}(r)$ for fixed $r \leqslant L_{c}$.

On the other hand, moments of the $f^{m}(r)$ are determined by the limit $Q \rightarrow 0$ in Eqs. (5.11) and (5.12). This gives

$$
\begin{aligned}
& \hat{f}_{1} \approx \frac{L_{c}^{2}}{\beta \sigma W^{2}}\left(1-Q^{2}+\cdots\right), \\
& \hat{f}_{2} \approx \frac{2 \pi L_{c}^{2}}{\left(2 \pi \beta \sigma W^{2}\right)^{2}}\left(1 / 2-Q^{2} / 12+\cdots\right) .
\end{aligned}
$$

Thus we have $f_{1,0}=L_{c}^{2} / \beta \sigma \mathrm{W}^{2} ; f_{1,2}=4 L_{c}^{2} f_{1,0}$ and $f_{2,0}$ $=\pi L_{c}^{2} /\left(2 \pi \beta \sigma W^{2}\right)^{2} ; f_{2,2}=2 / 3 L_{c}^{2} f_{2,0}$. These exact results will serve as a check on the approximations, valid for arbitrary $m$, that we now develop.

For fixed $q \gg L_{c}^{-1}$ (and hence as $L_{c} \rightarrow \infty$, any fixed $q>0$ ), only the behavior of $K_{0}$ at small arguments is important in Eq. (5.10). Equivalently, we can use Eq. (4.13) for $f(r)$ in Eq. (5.5) and find

$$
\begin{aligned}
\hat{f}_{m}(q) \approx & \int d \mathbf{r}\left[1-m \frac{\ln \left(r q_{\max }\right)}{\ln \left(L_{c} q_{\max }\right)}\right. \\
& \left.+O\left(\frac{r}{L_{c} \ln L_{c}}\right)\right] e^{-i q \cdot r} .
\end{aligned}
$$

The first term does not contribute for $q>0$. Thus the dominant term for $q \gg L_{c}^{-1}$ as $L_{c} \rightarrow \infty$ is

$$
\hat{f}_{m}(q) \approx \frac{2 \pi}{\ln \left(L_{c} q_{\max }\right)} \frac{m}{q^{2}}=\frac{1}{\beta \sigma W^{2}} \frac{m}{q^{2}},
$$

where we note that $1 / 2 \pi \ln r$ is the Green's function for the Laplace operator in two (interface) dimensions, ${ }^{29}$ with Fourier transform $q^{-2}$. Equation (5.16) for $m=1,2$ is in precise agreement with the exact results (5.13). Note that the dominant order result (5.16) is essentially independent of the cutoff $q_{\max }$.

In passing, we note that Eq. (5.16) can be derived in perhaps a more systematic way by substituting the integral representation ${ }^{30}$

$$
K_{0}(y)=\int_{0}^{\infty} d t e^{-y \cosh t}
$$

into Eq. (5.10), and carrying out the integration over $\mathbf{r}=(r, \theta)$. We find ${ }^{31}$

$$
\begin{aligned}
\hat{f}_{m}(q)= & \frac{2 \pi}{\left(2 \pi \beta \sigma W^{2}\right)^{m} q^{2}} \int_{0}^{\infty} d t_{1} \cdots \\
& \times \int_{0}^{\infty} d t_{m} \frac{\lambda B_{m}}{\left(1+\lambda^{2} B_{m}^{2}\right)^{3 / 2}},
\end{aligned}
$$

where

$$
B_{m} \equiv \cosh t_{1}+\cosh t_{2}+\cdots+\cosh t_{m}
$$

and

$$
\lambda \equiv Q^{-1}=\left(q L_{c}\right)^{-1} .
$$

Although Eq. (5.18) is exact, we have been able to carry out the remaining integrations exactly only for $m=1$ or 2 , yielding Eqs. (5.11) and (5.12). However, for finite $q$ as $L_{c} \rightarrow \infty$, we need only the limit as $\lambda \rightarrow 0$ in Eq. (5.18). This we can evaluate for general $m$. Defining $\widetilde{S} \equiv \lambda \sinh t_{m}$, we have from Eq. (5.19)

$$
\begin{aligned}
\lambda^{2} B_{m}^{2} & =\lambda^{2} B_{m-1}^{2}+2 \lambda^{2} B_{m-1} \cosh t_{m}+\lambda^{2} \cosh ^{2} t_{m} \\
& \approx \lambda^{2} B_{m-1}^{2}+\widetilde{S}^{2}+2 \lambda B_{m-1} \tilde{S}
\end{aligned}
$$

as $\lambda \rightarrow 0^{+}$. Using Eq. (5.21), the integration over $t_{m}$ in Eq. (5.18) yields ${ }^{32}$ for $q>0$

$$
\begin{aligned}
\hat{f}_{m}(q) \approx & \frac{2 \pi m}{\left(2 \pi \beta \sigma W^{2}\right)^{m} q^{2}} \int_{0}^{\infty} d t_{1} \cdots \\
& \times \int_{0}^{\infty} d t_{m-1}\left[1-\frac{\lambda B_{m-1}}{\left(1+\lambda^{2} B_{m-1}^{2}\right)^{1 / 2}}\right] .
\end{aligned}
$$

For small $\lambda$, because of the exponential behavior in Eq. (5.19) the integrand in Eq. (5.22) behaves like a step function, vanishing rapidly if any of the $t_{j}$ are greater than $\ln \lambda^{-1}$. As $\lambda \rightarrow 0^{+}$, one can show that this picture becomes increasingly accurate and the integration over all $t_{j}$ yields the volume of the hypercube each of whose sides is $\ln \lambda^{-1}$ long, i.e., $\ln ^{m-1}\left(\lambda^{-1}\right)$. Using Eq. (1.1), as $g \rightarrow 0^{+}$we again obtain the fundamental result (5.16).

Equation (5.16) allows us to evaluate $\widehat{C}_{\tau}$ in Eq. (5.9) to dominant order for finite $q \gg L_{c}{ }_{c}^{-1}$. We find

$\widehat{C}_{\tau}\left(\tau_{1}, \tau_{2}, q\right) \approx e^{1 / 2 \tau_{1}^{2}+1 / 2 \tau_{2}^{2}} \frac{2 \sqrt{\pi}}{(\Delta \rho)^{2}} \beta \sigma W^{2} q^{2} \delta\left(\tau_{1}-\tau_{2}\right)$,

or, from Eqs. (5.3), (2.9), and (2.6), for the usual direct correlation function

$$
\widehat{C}\left(z_{1}, z_{2}, q\right) \approx-\frac{\beta \sigma}{(\Delta \rho)} q^{2} \frac{\delta\left(z_{1}-z_{2}\right)}{\rho^{\prime}\left(z_{2}\right)} .
$$

Note that all values of $m$ in Eq. (5.9) contribute to yield Eq. (5.23) or (5.24).

Since Eq. (5.24) is valid for any fixed $q>0$ as $L_{c} \rightarrow \infty$, we can formally invert it and obtain for fixed $r_{12} \ll L_{c}$

$C\left(z_{1}, z_{2}, r_{12}\right) \approx \frac{\beta \sigma}{\Delta \rho} \nabla^{2} \delta\left(\mathbf{r}_{1}-\mathbf{r}_{2}\right) \frac{\delta\left(z_{1}-z_{2}\right)}{\rho^{\prime}\left(z_{2}\right)}$

This strongly suggests that the dominant term in $C$ is short ranged in both $z$ and $\mathbf{r}$ for $d=3$. Indeed Eq. (5.24) is identical to the dominant term for $d=2$ found by $\mathrm{BW}$ in their Eq. 
(7.3), when their result is expressed in unscaled variables.

Note that one cannot simply integrate Eq. (5.25) over $r_{12}$ to obtain interface moments of $C$, since this would miss contributions at very small $q<L_{c}^{-1}$, where Eq. (5.16) is not accurate. When moments are taken, lower order correction terms like the term of $O\left(W^{-2}\right)$ in Eq. (5.13b) can make nonnegligible contributions. However, when Eq. (5.23) or (5.24) is multiplied by $\rho^{\prime}\left(z_{2}\right)$ and integrated over $z_{2}$, we do recover the dominant term in the exact results $(3.7 b)$ or (3.8). Thus any lower-order correction terms to Eq. (5.23) or (5.24) must vanish when multiplied by $\rho^{\prime}\left(z_{2}\right)$ and integrated over $z_{2}$.

\section{APPROXIMATE EVALUATION OF MOMENTS OF $c$ FOR $d=3$}

Though the above calculations show that the dominant features of $C$ are the same in $d=3$ as in $d=2$, differences can be seen in the detailed behavior of interface moments. As mentioned above, these differences vanish when $C$ is projected onto $\rho^{\prime}(z)$ as in the TZ formula. Thus a calculation of the unprojected moments of $C$ is mainly of academic interest. However, it was her calculation of $C_{2}$, the second moment of $C$ in $d=3$, that lead Ciach to question the usual interpretation of the TZ formula. ${ }^{33}$

Moreover, as we shall see, the formal expression for $C_{2}$ yields a series in which each term individually diverges in the limit $L_{c} \rightarrow \infty$. This lead Requardt and Wagner ${ }^{2}$ to argue that $C$ is ill-defined and that the capillary wave model breaks down. It is therefore of interest to demonstrate that an explicit resummation of terms of this kind can lead to a sensible result.

To begin our calculation, we see from Eqs. (5.4) and (5.9) that the zeroth and second interface moments of $H$ and $C$ can immediately be obtained provided that we can calculate the moments of $[f(r)]^{m}$ in Eqs. (5.7) and (5.8) for arbitrary $m$. Thus, the basic integral we must calculate is

$$
I_{m}^{p} \equiv \int_{0}^{\infty} d x x^{p+1} K_{0}^{m}(x) .
$$

Unlike the previous calculation leading to Eq. (5.16), the behavior of $K_{0}(x)$ for all $x$ is required in Eq. (6.1). Since $K_{0}(x)$ vanishes exponentially for $x \gtrsim 1$ and diverges as $-\ln (x)$ for small $x$, the integrand is dominated by the small $x$ behavior for large $m$. A natural approximation therefore is

$$
K_{0}(x) \approx \begin{cases}\log (a / x), & 0 \leqslant x \leqslant x_{c} \\ 0, & x>x_{c}\end{cases}
$$

where $x_{c}$ is a constant of $O(1)$. The choice $a \equiv 2 e^{-\gamma_{E}}$ $\approx 1.1229$, where $\gamma_{E}$ is Euler's constant, reproduces the small argument behavior of $K_{0}(x)$ to $O\left(x^{2}\right)$. Table I compares the exact values of $I_{m}^{p}$ (calculated numerically) with the approximate result

$$
I_{m}^{p} \approx \frac{a^{p+2} m !}{(p+2)^{m+1}},
$$

calculated by substituting Eq. (6.2) into Eq. (6.1), using the choice $x_{c}=a$. We see that there are errors of $O(1)$ in the values $I_{m}^{p}$ for small $m$, but the relative error becomes in-

\begin{tabular}{|c|c|c|c|c|}
\hline \multicolumn{3}{|c|}{$p=0$} & \multicolumn{2}{|c|}{$p=2$} \\
\hline$m$ & "exact" & Eq. (6.3) & "exact" & Eq. (6.3) \\
\hline 1 & 1.0 & 0.315 & 4.0 & 0.099 \\
\hline 2 & 0.5 & 0.315 & 0.333 & 0.050 \\
\hline 3 & 0.586 & 0.473 & 0.115 & 0.037 \\
\hline 4 & 1.051 & 0.946 & 0.075 & 0.037 \\
\hline 5 & 2.49 & 2.36 & 0.074 & 0.047 \\
\hline 6 & 7.27 & 7.09 & 0.095 & 0.070 \\
\hline 7 & 25.0 & 24.8 & 0.150 & 0.122 \\
\hline 8 & 100 & 99.3 & 0.281 & 0.245 \\
\hline 10 & 2250 & 2234 & 1.47 & 1.38 \\
\hline 12 & & & 11.71 & 11.35 \\
\hline 14 & & & 131 & 129 \\
\hline 16 & & & 1950 & 1936 \\
\hline 18 & & & 37200 & 37034 \\
\hline
\end{tabular}

TABLE I. Values of $I_{m}^{p}$ in Eq. (6.1) calculated numerically and compared to Eq. (6.3) with $x_{c}=a=1.1229$.

creasingly small for large $m$, as one would expect. ${ }^{34}$

Using Eqs. (6.3), (5.10), and (4.10), the moments $f_{m, 0}$ and $f_{m, 2}$ in Eqs. (5.7) and (5.8) are approximated by

$$
f_{m, 0} \approx \frac{2 \pi L_{c}^{2} a^{2} m !}{2^{m+1} \ln ^{m}\left(L_{c} q_{\max }\right)}
$$

and

$$
f_{m, 2} \approx \frac{2 \pi L_{c}^{4} a^{4} m !}{4^{m+1} \ln ^{m}\left(L_{c} q_{\max }\right)} .
$$

Unlike the results (5.16) for $q>0$, which were asymptotically exact as $L_{c} \rightarrow \infty$, the errors in Eqs. (6.4) and (6.5) do not vanish in this limit. Thus any results using Eqs. (6.4) and (6.5) can be only of qualitative significance.

Nevertheless, we believe that they are useful approximations to illustrate that a proper summation of all terms in Eq. (5.9) with $g$ finite will lead to credible results. In particular, the approximations (6.4) and (6.5) possess the main feature that lead Requardt and Wagner to suspect that $C$ would not be well defined for $d=3$. Since each value of $m$ has a higher power of $\ln L_{c}$ in the denominator, in the limit $L_{c} \rightarrow \infty$ the expressions for the zeroth and second moments $H_{0}$ and $H_{2}$ derived from Eq. (5.4) are dominated by the lowest $(m=0)$ eigenfunction. However, this suggests that there could be trouble for the inverse functions, since if the coefficients of the higher eigenfunctions in $H$ vanished identically, the inverse function $C$ could not be defined at all. Another way to phrase this argument is to note that the expansion of $C_{2}$ derived from Eqs. (5.9), (6.4), and (6.5) is in powers of $\left(\ln L_{c}\right)^{m}$, which appears to diverge for large $L_{c}$ and $m$. See Eqs. (6.6) and (6.7) below.

However, we must keep $L_{c}$ finite while carrying out these sums. As we will see, $C_{0}$ and $C_{2}$ then are well defined. As is clear from Eqs. (6.4) and (6.5), the calculation of $C_{0}$ and $C_{2}$ involves very similar issues. Since the second moment has received the most attention, we now calculate it explicitly, using the approximations (6.4) and (6.5).

Formally expanding Eqs. (5.9) and (5.5) in powers of $q$, we find from the coefficient of the $q^{2}$ term that 


$$
\begin{aligned}
C_{\tau, 2}\left(\tau_{1}, \tau_{2}\right)= & -e^{1 / 2 \tau_{1}^{2}+1 / 2 \tau_{2}^{2}}(\Delta \rho)^{-2} 2 \sqrt{\pi} \\
& \times \sum_{m=0}^{\infty} \frac{(m+1) f_{m+1,2}}{f_{m+1,0}^{2}} \psi_{m}\left(\tau_{1}\right) \psi_{m}\left(\tau_{2}\right)
\end{aligned}
$$

Using the fact that

$$
\psi_{0}^{2}(\tau)=\pi^{-1 / 2} e^{-\tau^{2}}=(\Delta \rho)^{-1} d \rho_{s}(\tau) / d \tau,
$$

we see that the TZ formula (3.10) projects out the $m=0$ term in Eq. (6.6). It is satisfied as an identity when the exact results for $f_{1,2}$ and $f_{1,0}$ given after Eq. (5.14) are used along with Eq. (6.6).

Here, however, we want to study the properties of the unprojected $C_{\tau, 2}$. Using Eqs. (6.4) and (6.5), we can approximately rewrite Eq. (6.6) as

$$
\begin{aligned}
C_{\tau, 2}\left(\tau_{1}, \tau_{2}\right) \approx & -e^{1 / 2 \tau_{1}^{2}+1 / 2 \tau_{2}^{2}}(\Delta \rho)^{-2} 2 \sqrt{\pi} \beta \sigma W^{2} \\
& \times \sum_{m=0}^{\infty} \sum_{n=0}^{\infty} \delta_{m n} \frac{A^{m}}{\sqrt{m !}} \frac{A^{n}}{\sqrt{n !}} \psi_{m}\left(\tau_{1}\right) \psi_{n}\left(\tau_{2}\right),
\end{aligned}
$$

where

$$
A \equiv\left[\ln \left(L_{c} q_{\max }\right)\right]^{1 / 2},
$$

and $\delta_{m n}=1$ for $m=n$ and 0 otherwise.

Introducing the integral representation

$$
\delta_{m n}=\frac{1}{2 \pi} \int_{0}^{2 \pi} d y e^{i(m-n) y}
$$

into Eq. (6.7) and changing the order of summation and integration, we have the product of two independent sums, each of which can be evaluated by making use of the generating function for harmonic oscillator eigenfunctions ${ }^{35}$ :

$$
\sum_{n=0}^{\infty} \frac{B^{n}}{\sqrt{n !}} \psi_{n}(\tau)=\pi^{-1 / 4} e^{-1 / 2 \tau^{2}-1 / 2 B^{2}+\sqrt{2} \tau B} .
$$

Thus Eq. (6.7) can be written exactly as

$$
\begin{aligned}
C_{\tau, 2}\left(\tau_{1}, \tau_{2}\right) \approx & \frac{\beta \sigma W^{2}}{\pi(\Delta \rho)^{2}} \int_{0}^{2 \pi} d y \exp \left[-A^{2} \cos 2 y\right. \\
& \left.+\sqrt{2} A\left\{\tau_{+} \cos y+i \tau_{-} \sin y\right\}\right],
\end{aligned}
$$

where $\tau_{ \pm} \equiv \tau_{1} \pm \tau_{2}$.

Using Eq. (5.3), the usual second moment can then be written, with $y_{1} \equiv \pi / 2-y$,

$$
\begin{aligned}
C_{2}\left(z_{1}, z_{2}\right) \approx & \frac{\beta \sigma}{2 \pi(\Delta \rho)^{2}} \int_{-\pi / 2}^{\pi / 2} d y_{1} \exp \left[A^{2} \cos 2 y_{1}\right. \\
& \left.+\alpha z_{+} \sin y_{1}\right] \cos \left(\alpha z_{-} \cos y_{1}\right),
\end{aligned}
$$

where

$$
z_{ \pm} \equiv z_{1} \pm z_{2}
$$

and

$$
\alpha \equiv(2 \pi \beta \sigma)^{1 / 2} \text {. }
$$

For $z_{1}=z_{2}=0$, the integral in Eq. (6.12) gives

$$
\begin{aligned}
C_{2}(0,0) & \approx \frac{\beta \sigma}{2(\Delta \rho)^{2}} I_{0}\left(A^{2}\right), \\
& \approx \frac{\beta \sigma}{2(\Delta \rho)^{2}} \frac{\left(L_{c} q_{\max }\right)}{\left[2 \pi \ln \left(L_{c} q_{\max }\right)\right]^{1 / 2}},
\end{aligned}
$$

where $I_{0}$ is the modified Bessel function of the first kind, and Eq. (6.15b) holds for large $L_{c}$.

For general $z_{1}, z_{2} \ll W$, the integrand in Eq. (6.12) is dominated by values of $y_{1}$ near zero as $L_{c} \rightarrow \infty$. Expanding to second order, we obtain the approximate result

$$
C_{2}\left(z_{1}, z_{2}\right) \approx \frac{\beta \sigma \cos \left(\alpha z_{-}\right)\left(L_{c} q_{\max }\right)}{2(\Delta \rho)^{2}\left[2 \pi \ln \left(L_{c} q_{\max }\right)\right]^{1 / 2}},
$$

which agrees with $(6.15 \mathrm{~b})$ for $z_{-}=0$.

These approximate results suggest that $C_{2}\left(z_{1}, z_{2}\right)$ has an oscillatory component of large amplitude, proportional to $L_{c} q_{\max }$, that varies on the length scale $\alpha^{-1}$ as a function of $z_{\ldots} \cdot{ }^{36}$ The fact that the amplitude in Eq. (6.16) is proportional to $L_{c}$ causes no problems for any finite $g>0$, and indeed, as one would expect on general grounds, the divergence in (6.16) is much less strong than that obtained from the corresponding expression for $H_{2}$, which diverges as $L_{c}^{4}$.

However, since there is an important and nontrivial dependent on the cutoff $q_{\max }$ in Eqs. (6.15b) and (6.16), these results are certainly nonuniversal. Their detailed behavior is probably an artifact of the sharp cutoff used in Eq. (2.1), as well as our use of the approximate expressions (6.4) and (6.5).

Nevertheless, we expect that some general features of Eq. (6.16) would be seen in the detailed expressions for $C_{2}$ obtained from a variety of models in $d=3$ with different treatments of the cutoff. It is clear from Eq. (5.9) or (6.6) that in general there will be correction terms to the dominant $\delta$ function behavior, which is exact ${ }^{6}$ only for $d=2$. Such corrections arise from the term of $O\left(W^{-2}\right)$ in Eq. (5.13b) and similar terms for $m>2$ in the evaluation of $\hat{f}_{m}(q)$. While negligible for fixed $r_{12} \ll L_{c}$ and $z_{1}, z_{2} \ll W$ in $C\left(z_{1}, z_{2}, r_{12}\right)$, this additional structure can become noticeable when interface moments are taken. While these corrections can lead to formal divergences in $C_{2}$ as $L_{c} \rightarrow \infty$, they must be much less strong than the well-understood divergences found in $\mathrm{H}_{2}$, since the latter arise from the dominant order terms. Further, we expect oscillatory behavior in $C_{2}$ on the microscopic scale of $O\left(\xi_{B}\right)$ from the correction terms, since we know that on projection onto the smooth function $\rho^{\prime}(z)$ as in Eq. (3.7) or (3.8), the contributions from the correction terms vanish. Indeed, using Widom's hyperscaling relation ${ }^{1,7}$ in $d=3$, we see the length scale $\alpha^{-1}$ in Eq. (6.14) is proportional to $\xi_{B}$.

\section{FINAL REMARKS}

In this paper we have analyzed in some detail the capillary wave predictions for the direct correlation function $C$ in $d=3$. We conclude that the resulting expressions are wellbehaved provided one uses an arbitrarily small but nonzero external field to induce phase separation and to localize the average interface position. The presence of such a field is also necessary to ensure that there is an invertible relation between the density and the field, as required in the density functional approach ${ }^{8,9,12}$ that leads to the TriezenbergZwanzig formula. ${ }^{3}$

We see no reason to doubt ${ }^{1,2}$ the correctness of the capillary wave description of long wavelength interface fluctu- 
ations on the basis of its prediction in $d=3$. On the contrary, those predictions that follow solely from the long wavelength structure of the interface Hamiltonian should be universally valid and model independent. This holds in particular for the logarithmic divergence of the interface width in $d=3$, a special case of the prediction that the height difference correlation function $G(r)$ in $\mathrm{Eq}$. (2.5) is proportional to $\ln r$ for $\xi_{B} \ll r \ll L_{c} .^{5,14}$ This agrees with exact results ${ }^{11}$ for $T \geqslant T_{R}$ in van Beijeren's BCSOS interface model, which has a different treatment of the short distance cutoff. It is hard for us to imagine how such a lattice model could be rough with the continuum liquid-vapor interface width remaining finite. ${ }^{2}$ Although $d=3$ is a crossover dimension in capillary wave theory, its prediction of a logarithmically divergent interface width should be quite robust.

Other general predictions of the capillary wave model include the existence of long-ranged correlations along the interface in the pair correlation function $H^{5,20}$ The direct correlation function $C$, on the other hand, is defined as the inverse of $H$, and therefore, in general, has some features that depend sensitively on the short-ranged properties of the particular interface model considered. Even in $d=2$, where the capillary wave model yields exact $\delta$ function behavior for $C$ to dominant order, ${ }^{6}$ other models with different treatments of the short distance behavior ${ }^{24}$ will have additional shortranged structure in $C$. As a result, it is difficult to gain an intuitive understanding of all the detailed features of $C$.

However, the projected part of $C$ in Eq. (3.7) or (3.8) that enters the TZ formula is model independent. We have shown elsewhere ${ }^{12}$ how this form can be justified physically and how it can be related to standard mean-field expressions ${ }^{7}$ in a small system where long wavelength fluctuations are suppressed.

The logarithmic divergence of the equilibrium interface width in $d=3$ is, of course, very hard to test experimentally. Nevertheless, many other aspects of capillary wave theory have been probed experimentally, both with light scattering and by $x$-ray reflectivity measurements. ${ }^{23,37,38}$ Likewise, a variety of other interfacial phenomena associated with wetting, roughening, random systems, etc., can be understood by using a long wavelength interface Hamiltonian similar to the capillary wave Hamiltonian. ${ }^{10,11}$

Another implication of this viewpoint concerns the nature of TZ formula (3.10) for the surface tension. In our interpretation, the main value of this formula and the related one derived by Wertheim ${ }^{20}$ is that these expressions and their derivation provide important insight into interface structure, both on short and on long length scales. ${ }^{12}$ Thus they provide important consistency checks for a given model system and for approximate theories of interface structure. On the other hand, the Kirkwood-Buff formula, ${ }^{7}$ which involves the short-ranged intermolecular potential, is the preferred formula to calculate the numerical value of the surface tension in simulations, precisely because it is essentially unaffected by the long wavelength fluctuations that play such an important role in determining the large scale structure of the interface.

There is a useful general analogy between the behavior of the interface pair correlation function as $g \rightarrow 0^{+}$and the bulk pair correlation function as $T \rightarrow T_{c} \cdot{ }^{14}$ The length scale on which exponential decay in $H$ occurs is set by the (diverging) capillary length in the former and the (diverging) bulk correlation length in the latter. This picture suggests a simple scaling ansatz relating correlations along the interface to the behavior of the interface width that indeed is satisfied by the capillary wave model correlation functions. ${ }^{6,14}$

However, this analogy does not imply that the detailed behavior of the interface pair correlation function can be mapped onto that of the bulk pair correlation function near the critical point in $d^{\prime} \equiv d-1$ dimensions. The essential difference is that in capillary wave theory the long wavelength "stiffness constant" $\sigma$ (i.e., the coefficient of the $\nabla^{2}$ term) approaches a finite limit as $g \rightarrow 0^{+}$, while in the bulk the analogous stiffness gets renormalized to zero in the long wavelength limit as $T \rightarrow T_{c}{ }^{39}$ A dramatic manifestation of these differences is that the interface pair correlation function decays more slowly than that of the analogous bulk system with $\xi_{B}=L_{c}$, since for $d \leqslant 3$, the interface correlation function is $O(1)$ until $r$ is $O\left(L_{c}\right)$, while the magnitude of the bulk correlation function is very much smaller for $r$ of $O\left(\xi_{B}\right)$ because of the $r^{-\left(d^{\prime}-2+\eta\right)}$ power law decay occurring for $r \ll \xi_{B} .^{7}$

This difference, which technically reflects the nonGaussian nature of the bulk fixed point for $d<4,,^{39}$ can perhaps be understood physically by considering the very different nature of the density fluctuations leading to long-ranged correlations in the two cases. In the bulk, correlations can be thought of as arising from "droplet" excitations, when two points separated by a distance $r$ are found within the same droplet. As $T \rightarrow T_{c}$, the surface tension becomes increasingly small and a picture of isolated compact droplets breaks down. Rather we have droplets within droplets, and they are better thought of as fractals rather than compact objects. ${ }^{40}$ This complex behavior on all length scales up to $O\left(\xi_{B}\right)$ leads to a more rapid decay of correlations that manifests itself in the existence of a nonzero value for the exponent $\eta$.

The interfacial fluctuations that build up the correlations in $H$ with $\sigma$ finite as $g \rightarrow 0^{+}$for $r \ll L_{c}$ (and $z_{1}, z_{2} \ll W$ ) are much simpler, however. On scales $q_{\max }^{-1} \ll r \ll L_{c}$, the relevant fluctuations are nearly vertical displacements of the interface that are coherent over a distance $r$. On this scale, the interface is essentially sharp and single valued (Euclidean instead of fractal) and as a result, no anomalous powers analogous to having $\eta>0$ arise. Thus capillary wave theory has none of the complications of bulk critical phenomena for $d<4$, provided that one avoids the ambiguities arising from being exactly at the "critical point" $g \equiv 0$.

\section{ACKNOWLEDGMENT}

We are grateful to D. A. Huse for helpful discussions.

\footnotetext{
'A. Ciach, Phys. Rev. A 36, 3990 (1987).

${ }^{2}$ M. Requardt and H. J. Wagner (preprint).

${ }^{3}$ D. G. Triezenberg and R. Zwanzig, Phys. Rev. Lett. 28, 1183 (1972).

${ }^{4}$ F. P. Buff, R. A. Lovett, and F. H. Stillinger, Phys. Rev. Lett. 15, 621 (1965).

${ }^{5}$ J. D. Weeks, J. Chem. Phys. 67, 3106 (1977).

${ }^{6}$ D. Bedeaux and J. D. Weeks, J. Chem. Phys. 82, 972 (1985).
} 
${ }^{7}$ For a recent review, see J. S. Rowlinson and B. Widom, Molecular Theory of Capillarity (Clarendon, Oxford, 1982).

${ }^{8}$ See, for example, G. Stell, in The Equilibrium Theory of Classical Fluids, edited by H. L. Frisch and J. L. Lebowitz (Benjamin, New York, 1965), p. II-171; J. K. Percus, ibid. p. II-33; an elementary introduction is given in Sec. 7.5 of R. Balescu, Equilibrium and Non-equilibrium Statistical Mechanics (Wiley, New York, 1975).

${ }^{9}$ R. Lovett, C. Y. Mou, and F. P. Buff, J. Chem. Phys. 65, 570 (1976).

${ }^{10}$ See, for example, M. E. Fisher, J. Chem. Soc. Faraday Trans. 2 82, 1569 (1986).

${ }^{1}$ See, for example, J. D. Weeks, in Ordering in Strongly Fluctuating Condensed Matter Systems, edited by T. Riste (Plenum, New York, 1980), p. 293.

${ }^{12}$ J. D. Weeks and W. van Saarloos, J. Phys. Chem. 93, 6969 (1989).

${ }^{13}$ In a real liquid-vapor system it is convenient to consider a "truncated" field (Ref. 14) so that the bulk phase properties are not strongly affected by the field. In the capillary wave model, the bulk phases are trivial and this procedure is not necessary.

${ }^{14}$ J. D. Weeks, Phys. Rev. Lett. 52, 2160 (1984).

15J. D. Weeks, D. Bedeaux, and B. J. A. Zielinska, J. Chem. Phys. 80,3790 (1984).

${ }^{16} \mathrm{G}$. Gallavotti, Riv. Nuovo Cimento 2, 133 (1972).

${ }^{17}$ R. L. Dobrushin, Theory Probab. Its Appl. (USSR) 17, 619 (1972).

${ }^{18}$ D. A. Huse, W. van Saarloos, and J. D. Weeks, Phys. Rev. B 32, 233 (1985).

${ }^{19}$ See M. P. Gelfand and M. E. Fisher, Int. J. Thermophys. 9, 713 (1988) for an excellent discussion of this point.

${ }^{20}$ M. S. Wertheim, J. Chem. Phys. 65, 2377 (1976).

${ }^{21}$ See, for example, D. Jasnow, Rep. Prog. Phys. 47, 1059 (1984).

${ }^{22}$ R. F. Kayser, Phys. Rev. A 33, 1948 (1986). Perhaps the first appearance of this idea can be found in F. H. Stillinger, J. Chem. Phys. 47, 2513 (1967).

${ }^{23}$ J. V. Sengers and J. M. J. van Leeuwen, Phys. Rev. A 39, 6346 (1989); J. M. J. van Leeuwen and J. V. Sengers, Physica A 157, 839 (1989).

${ }^{24}$ See, for example, results for the direct correlation function for the $d=2$ solid-on-solid model interface calculated by J. Stecki, A. Ciach, and J. Dudowicz, Phys. Rev. Lett. 56, 1482 (1986). See also P. C. Hemmer and B. Lund, J. Phys: A 21, 3463 (1988).
${ }^{25}$ A. Ciach, J. Dudowicz, and J. Stecki, Physica A 145, 327 (1987) and references therein.

${ }^{26}$ We have not examined the crossover behavior as $d \rightarrow 3$.

${ }^{27}$ I. S. Gradshteyn and I.M. Ryzhik, Table of Integrals, Series, and Products (Academic, New York, 1965), Eq. 6.521.2.

${ }^{28}$ A. Prudnikov, Yu. A. Bryckov, and O. I. Marichev, Integrals and Series (Gordon and Breach, New York, 1986), Vol. 2, Eq. 2.16.43.2.

${ }^{29}$ See, for example, P. M. Morse and H. Feshbach, Methods of Theoretical Physics (McGraw-Hill, New York, 1953), Vol. 1, p. 798.

${ }^{30}$ Reference 27, Eq. 8.432.1.

${ }^{31}$ The integration over $r$ can be done using Ref. 27, Eq. 3.944.12, and over $\theta$ using Ref. 27, Eq. 3.613.1.

${ }^{32}$ The integration can be carried out by charging variables to $\widetilde{S}$ and using Ref. 27, Eq. 2.264.5.

${ }^{33}$ We believe that Ciach's calculation is incorrect, mainly because she took an approximation for $P\left(z_{1}, z_{2}, r_{12}\right)$ in Eq. (2.7) that is valid only for $r<L_{c}$, and integrated it over all $r$, thus obtaining an incorrect expression for the second moment. In addition, we have not been able to justify some of the subsequent steps of her analysis in which $C_{2}$ is obtained through Fourier inversion.

${ }^{34}$ Note that if one were to take a different cutoff $x_{\mathrm{c}}$ of $O(1)$ in Eq. (6.2), the large $m$ approximation would not be affected, but the results for small $m$ would. Since $K_{0}(x)>\ln (a / x)$, it is easy to see that the choice $x_{c}=a$ made in Eq. (6.2) yields the best approximation for $m=1$ and, indeed, for all odd $m$.

${ }^{35}$ See, for example, A. Messiah, Quantum Mechanics (North-Holland, New York, 1965), p. 491.

${ }^{36}$ This resembles some results found by Ciach (Ref. 1) though we have a very different interpretation.

${ }^{37}$ See E. Mazur and D. S. Chung, Physica A 147, 387 (1987) and references therein. See also J. Meunier, J. Phys. (Paris) 48, 1819 (1987).

${ }^{38}$ A. Braslau, P. S. Pershan, G. Swislow, B. M. Ocko, and J. Als-Nielsen, Phys. Rev. A 38, 2457 (1988).

${ }^{39}$ See, for example, S. K. Ma, Modern Theory of Critical Phenomena (W. A. Benjamin, Reading, MA, 1976).

${ }^{40}$ See, for example, A. D. Bruce, Physica B 127, 180 (1984) and references therein; A. Coniglio, Phys. Rev. Lett. 62, 3054 (1989). 